\title{
Correlation of Microstructure and Mechanical Properties in Nuclear Reactor Stainless Steels
}

\author{
D. Scotto D'Antuono*, G. Vetterick*, M. Kirk**, and M.L. Taheri* \\ *Department of Materials Science and Engineering, Drexel University, Philadelphia, PA \\ **IVEM-Tandem Facility, Argonne National Laboratory, Argonne, IL
}

Stainless steels used in nuclear reactors experience extreme conditions during operation, requiring microstructures capable of withstanding temperature, stress, and irradiation without experiencing negative effects. One detrimental result of these extreme conditions is radiation induced segregation (RIS), which causes a redistribution of the atomic structure of the material, most notably at grain boundaries. This can lead to a change in the mechanical properties of the materials, such as grain boundary embrittlement [1].

Much research has been performed to examine the effects of RIS on austenitic steels. It has been shown that chromium is known to deplete at grain boundaries at high temperatures during irradiation while the nickel concentration increased [2]. However, the direct effects of solute segregation at the grain boundaries has yet to be accurately demonstrated and is essential to producing better materials. The results of this study will provide a more concrete understanding of the solute movement depending on the grain boundary type and density within the sample. For instance, special grain boundaries known as coincidence site lattice (CSL) boundaries, shown in Fig. 1, have a high level of atomic fit and are proposed to reduce the effects of RIS. Confirmation of this and similar effects will allow the use of grain boundary engineering to reduce RIS and create a more dependable material.

Stainless steel samples of two different compositions (304 and 316L) from Carpenter Technology Corporation will be analyzed using various microscopy techniques. Electron Backscattered Diffraction (EBSD) will be used to show the grain orientation of the samples as well as help show the density of coincidence site lattice (CSL) boundaries. Samples containing specific densities of CSL boundaries will then be prepared for in situ ion beam irradiation and tensile tests at Argonne National Laboratories (Fig. 2). This tool will allow for proton irradiation as well as heated tensile testing simultaneously, mimicking the environment stainless steels experience during use in a light water reactor (LWR). The data collected from these experiments will be used to develop an understanding of the role that microstructure plays in improving material properties, such as creep resistance, of nuclear reactor stainless steels.

\section{References}

[1] T. R. Allen et al., Journal of Nuclear Materials. 255 (1997) 44-58.

[2] R. D. Carter et al., Journal of Nuclear Materials. 205 (1993). 361-373.

[3] M.A. Kirk et al., Microscopy Research and Technique. 72.5 (2009). 182-186

[4] This research was made possible by use of the Centralized Research Facilities in the College of Engineering at Drexel University.

[5] The IVEM-Tandem Facility at Argonne National Laboratory is a national user facility supported by the U.S. Dept. of Energy and we thank their staff for assistance with the ion irradiations. 


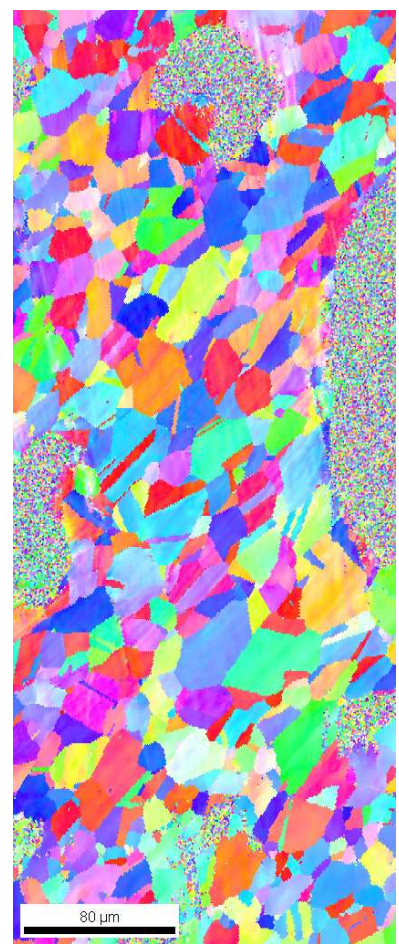

FIG. 1. Inverse pole figure revealing grain boundary orientations.

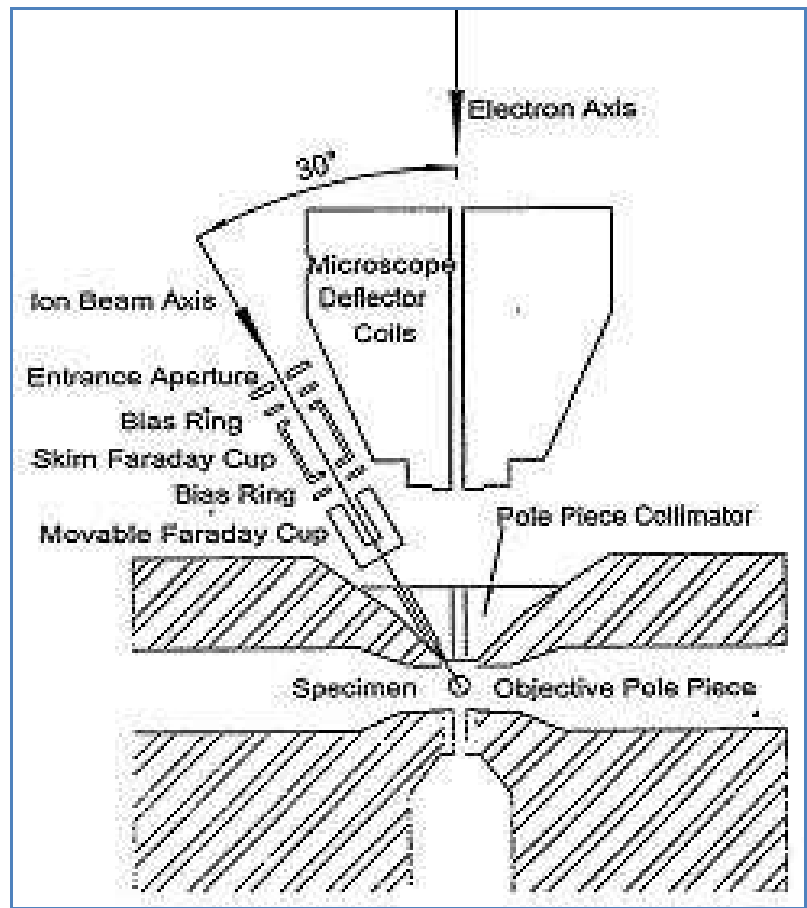

FIG. 2. Schematic of in situ Ion Beam TEM at Argonne National Laboratory [3]. 